\title{
ASS in der Primär- und Sekundärprävention kardiovaskulärer Erkrankungen
}

Alexander Hach, Reinhard Sauter, Harald Langer

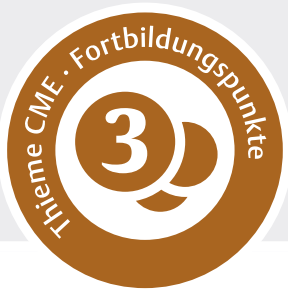

Der Thrombozytenaggregationshemmer Acetylsalicylsäure (ASS) ist das

Medikament der Wahl in der Sekundärprophylaxe zahlreicher kardiovaskulärer Erkrankungen [1]. Hingegen ist die Relevanz von ASS in der Primärprophylaxe nicht umfassend geklärt. Dieser Review beschäftigt sich mit den Therapieoptionen von ASS in der Primär- und Sekundärprävention.

\section{Einleitung}

Das bereits im Jahr 1899 durch die Bayer AG patentierte Medikament Aspirin wird seit nunmehr knapp 120 Jahren erfolgreich in der Medizin eingesetzt [2]. ASS wird pharmakologisch der Gruppe der nicht steroidalen Antirheumatika zugeordnet. Es wirkt antipyretisch, antiinflammatorisch, analgetisch sowie antithrombotisch als Thrombozytenaggregationshemmer. Abhängig von der Indikationsstellung kann es sowohl peroral (p. o.) als auch intravenös (i.v.) verabreicht werden [3].

Im Rahmen einer Einnahme als Thrombozytenaggregationshemmer ist in der Regel eine Dosierung von 75$100 \mathrm{mg} / \mathrm{d}$ ausreichend, während für eine Analgesie eine Dosis von 500-1000 mg benötigt wird [4]. Der am besten charakterisierte molekulare Wirkmechanismus von ASS als Thrombozytenaggregationshemmer ist die irreversible Inaktivierung der Prostaglandin-G/H-Synthase 1 (Cyclooxygenase 1, COX-1) [4,5]. Da die COX-1-Hemmung irreversibel ist, dauert die Wirkung von ASS bis zum Ende der Thrombozytenlebensdauer (ca. 8-12 d) an.

Die Inaktivierung von COX-1 durch niedrig dosiertes ASS führt zu einer lang anhaltenden Suppression der Thromboxan-(TX-)A2-Produktion und somit zur Hemmung der TXA2-vermittelten Aktivierung und Aggregation der Thrombozyten ( $\triangleright$ Abb. 1) [5-7].

Es wurden weitere Wirkungsmechanismen publiziert, welche die weiteren positiven Effekte von ASS aufklären sollen, wie z. B. die Prävention von venösen Thromboembolien [8], die Chemoprävention von Kolonkarzinomen und anderen malignen Erkrankungen [9] und die Verringerung des Demenzrisikos [10]. Zu diesen Mechanismen gehören die Acetylierung anderer Proteine, die an der Blutgerinnung beteiligt sind [8], die Hemmung der COX2-Aktivität [10] und andere COX-unabhängige Mechanismen [5].

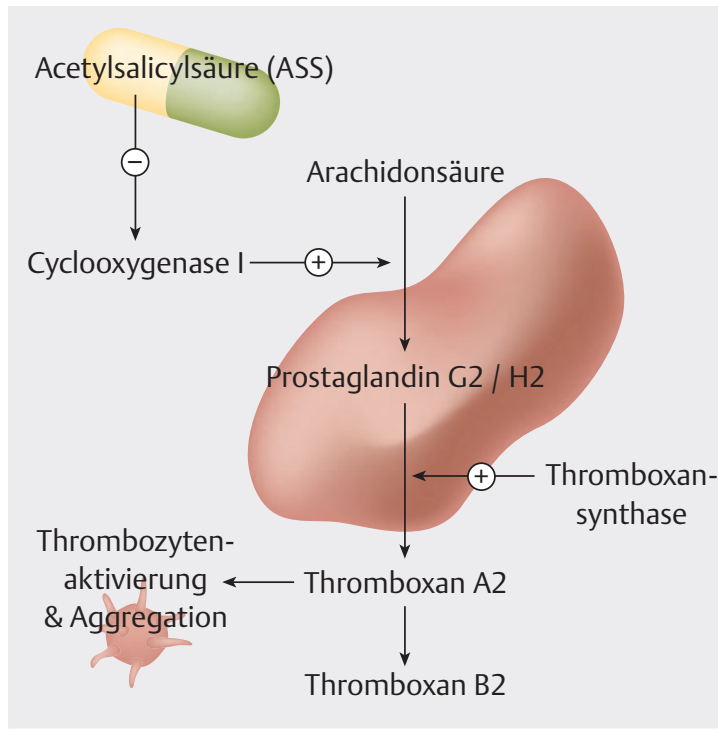

Abb. 1 Wirkmechanismus des Thrombozytenaggregationshemmers ASS.

Neben den therapeutischen Effekten zeigen fast 25\% der Personen, die mit nicht steroidalen Antirheumatika behandelt werden, verschiedenartige Nebenwirkungen, wie z.B. gastrointestinale Funktionsstörungen [11]. Als weitere Nebenwirkungen der ASS-Therapie können Übelkeit, Erbrechen und Sodbrennen auftreten. Bei bestehendem Asthma bronchiale kann der Gebrauch von ASS zu einer erhöhten Anfallsrate führen, da durch die Hemmung der Cyclooxygenasen vermehrt Arachidonsäure anfällt, welche in einer vermehrten Leukotrienbildung resultiert, was wiederum eine Bronchokonstriktion bewirkt [12]. Bei Kindern und Jugendlichen ist ASS kontraindiziert, da es das tödliche Reye-Syndrom auslösen kann [13]. Weitere Kontraindikationen leiten sich aus dem Nebenwirkungsspektrum ab. Bei bekannten Magen-DarmUlzera, bei Schwangerschaft im letzten Trimenon sowie 
bei einer hämorrhagischen Diathese sollte keine Therapie mit ASS erfolgen.

In einer Studie im Vereinigten Königreich wurde publiziert, dass ASS für über $60 \%$ der durch Arzneinebenwirkungen verursachten Todesfälle verantwortlich ist [14]. Die therapeutischen Wirkungen von ASS werden durch COX-2-Hemmung erzielt, jedoch führt die COX-1-Hemmung zu Nebenwirkungen [15]. Kreuzreaktionen können mit anderen nicht steroidalen Antirheumatika wie beispielsweise Ibuprofen, Diclofenac oder Naproxen auftreten.

Die Leitlinien der Europäischen Gesellschaft für Kardiologie (ESC) raten bei Patienten mit stabiler KHK zur täglichen Einnahme von ASS in niedriger Dosierung [16]. Üblich ist hierzulande eine Dosierung von $100 \mathrm{mg}$. In den USA wird üblicherweise mit $80 \mathrm{mg}$ ASS behandelt, in anderen Ländern kommen auch 160 mg ASS zum Einsatz. Bei einer ASS-Intoleranz wird in der ESC-Leitlinie alternativ zu Clopidogrel geraten.

\section{ASS in der Primärprävention}

Der Nutzen einer dauerhaften täglichen Dosis von $100 \mathrm{mg}$ ASS nach einem Myokardinfarkt oder ischämischen Schlaganfall zur Sekundär- beziehungsweise Rezidivprophylaxe ist bereits durch mehrere randomisierte klinische Studien belegt. Hierbei zeigt sich, dass der Nutzen in der Sekundärprophylaxe verglichen mit den $\mathrm{Ne}$ benwirkungen überwiegt [1]

Allerdings ist der Einsatz von ASS in der Primärprophylaxe hinsichtlich einer potenziellen protektiven Wirkung gegenüber kardiovaskulären Ereignissen nicht ausreichend geklärt.

Entstehung von Atherosklerose ist einer der Hauptrisikofaktoren für chronische Herzkrankheiten und zerebrovaskuläre Erkrankungen. Arterielle Thromben, welche aus rupturierten atherosklerotischen Plaques oder aus endothelialen Erosionen entstehen, sind meist Ursprung eines Herzinfarkts oder Schlaganfalls [1]. Thrombozyten sind ein wichtiger Bestandteil arterieller Thromben und spielen im Rahmen der Plaquebildung eine wichtige Rolle.
In diesem Artikel soll zunächst die Differenzierung zwischen Primär- und Sekundärprävention beleuchtet werden:

- Primärprävention bezieht sich auf den Erhalt der Gesundheit und der Vorbeugung von Krankheiten, bevor ein schädliches Ereignis oder eine Krankheit auftritt.

- Bei der Sekundärprävention sind bereits eine Krankheit, Risikofaktoren oder ein schädliches Ereignis vorhanden beziehungsweise geschehen und Ziel ist, die Früherkennung beziehungsweise das Voranschreiten der Krankheit zu verhindern.

Im Rahmen der Studien wurden Patienten je nach Studiendesign mit verschiedenen Vorerkrankungen (wie beispielsweise Diabetes) oder Risikofaktoren (hohes Alter), jedoch ohne schwerwiegendes kardiovaskuläres Ereignis eingeschlossen. Dementsprechend werden sie der Primärprävention zugeordnet, da es sich um eine Vorbeugung eines schädlichen Ereignisses handelt.

Mehrere Studien haben sich mit dem Nutzen von ASS in der Primärprävention zur Verhinderung von kardiovaskulären Ereignissen befasst: Die ASCEND-Studie, die ARRIVE-Studie und die ASPREE-Studie ( $\bullet$ Tab. 1$)$. Es wurde der Effekt einer langjährigen ASS-Gabe von $100 \mathrm{mg} / \mathrm{d}$ bei allen Studien mit unterschiedlichen Patientengruppen hinsichtlich kardiovaskulärer Ereignisse oder anderer Erkrankungen untersucht.

Merke
Der Nutzen von ASS in einer Dosis von $100 \mathrm{mg} / \mathrm{d}$
im Rahmen der Primärprävention wurde in
verschiedenen Studien untersucht.

Im Folgenden werden die verschiedenen Studien und ihre Ergebnisse kurz erklärt.

\section{Patienten mit Diabetes - ASCEND-Studie}

In der ASCEND-Studie („A study of Cardiovascular Events in Diabetes“) wurden 15840 Patienten mit Diabetes mellitus (jedwelcher Genese) und einem Mindestalter von 40 Jahren eingeschlossen. Die placebokontrollierte randomisierte Studie beinhaltet eine tägliche Gabe von ASS $100 \mathrm{mg} / \mathrm{d}$ bei einer durchschnittlichen Follow-up-Periode von 7,4 Jahren [17]. Patienten mit kardiovaskulären Vorerkrankungen wurden aus der Studie ausgeschlossen.

- Tab. 1 Übersicht Studien zu ASS in Primärprävention.

\begin{tabular}{|l|l|l|l|l|}
\hline Studie & Risiko & ASS-Dosis & Dauer & Patientenanzahl \\
\hline ASCEND & Diabetes & $100 \mathrm{mg} / \mathrm{d}$ & 7,4 Jahre & 12546 \\
\hline ARRIVE & mittleres kardiovaskuläres Risiko & $100 \mathrm{mg} / \mathrm{d}$ & 7,4 Jahre & 15840 \\
\hline ASPREE & hohes Alter $(\geq 70$ Jahre $)$ & $100 \mathrm{mg} / \mathrm{d}$ & 4 Jahre & 19114 \\
\hline
\end{tabular}


Primäre Endpunkte der ASCEND-Studie wurden definiert als nicht tödlicher Herzinfarkt, nicht tödlicher Schlaganfall oder transischämische Attacke sowie Tod durch ein vaskuläres Ereignis, welches nicht auf einer intrakraniellen Blutung basierte.

Die sekundären Endpunkte beinhalteten das Auftreten von gastrointestinalen Tumoren, welche innerhalb der ersten 3 Jahre des Follow-ups aufgetreten sein mussten, sowie jegliche Form eines schwerwiegenden vaskulären Ereignisses oder einer arteriellen Revaskularisierungsmaßnahme.

Im Patientenkollektiv, das mit täglich 100 mg ASS behandelt wurde, war gegenüber der Placebogruppe das Risiko für ein vaskuläres Ereignis, wie einen Herzinfarkt, Schlaganfall oder den Tod zu erleiden, um 12\% gesenkt (RR: 0,88; 95\%-KI 0,79-0,97; 8,5\% vs. 9,6\%; $p<0,01$ ) [17].

Diesem als moderat zu bezeichnenden Nutzen steht jedoch ein erhöhtes Blutungsrisiko entgegen: Mit der regelmäßigen ASS-Einnahme stieg das Blutungsrisiko um $29 \%$ an $(4,1$ vs. $3,2 \%, p=0,003)$. Die meisten Blutungen waren gastrointestinal (41,3\%), davon $62,3 \%$ im oberen und 32,9\% im unteren Gastrointestinaltrakt. Weitere Blutungslokalisationen waren Einblutungen im Auge (21,1\%) sowie intrakranielle Blutungen (17,2\%).

Die Autoren der Studien konsultieren, dass sich die positiven Effekte einer Primärprävention mit ASS 100 mg/d mit den negativen Effekten bei Diabetikern ausgleichen. Des Weiteren sind die Autoren der Meinung, dass Diabetiker bereits gut mit Statinen [18], Blutdrucksenkern und Antidiabetika eingestellt sind, sodass eine Hinzunahme von ASS keinen relevanten Nutzen bringen würde [17]. Die Adhärenz der Tabletteneinnahme sank mit zunehmender Studiendauer. Die Autoren mutmaßen, dass dadurch der positive Effekt der primärprophylaktischen ASS-Einnahme vermindert wurde [18].

Es wird in der Studie publiziert, dass die „Number needed to treat" mit ASS $100 \mathrm{mg}$, welche erforderlich ist, um ein schwerwiegendes vaskuläres Ereignis abzuwenden (definiert als nicht tödlicher Herzinfarkt oder Schlaganfall sowie eine transischämische Attacke oder Tod), 91 Patienten umfassen müsste. Jedoch würden bei dieser Dosierung 112 Patienten eine relevante Blutung erleiden [17].

Die Autoren der Studie diskutieren, dass vor allem bei älteren Menschen das individuelle Blutungsrisiko in die Entscheidungsfindung miteinbezogen werden müsse. Nach den Leitlinienempfehlungen der ADA (American Diabetes Association) und ACC (American College of Cardiology)/ AHA (American Heart Association) könnte ASS 100 mg/d bei Patienten mit einem hohen Risiko für arteriosklerotische kardiovaskuläre Erkrankungen (ASCVD) bei einem geringen Blutungsrisiko eingesetzt werden (COR IIB LOE
A) [18]. Bei Diabetespatienten mit einem niedrigen Risiko für kardiovaskuläre Erkrankungen überwiegt das Risiko, eine schwere Blutung zu erleiden, jedoch die positiven Effekte einer Primärprophylaxe.

\section{Merke \\ Aspirin kann bei Diabetikern ohne kardiovaskuläre Vorerkrankung nur marginal Herzinfarkten und Schlaganfällen vorbeugen bei einem erhöhten Risiko für das Auftreten starker Blutungen. \\ Patienten mit einem erhöhten kardiovaskulären Risiko - ARRIVE-Studie}

Bei der ARRIVE-Studie („Aspirin to reduce risk of initial vascular event") [19] handelt es sich um eine randomisierte, doppelblinde, placebokontrollierte, multizentrische Studie, welche in 7 Ländern (Deutschland, Italien, Irland, Polen, Spanien, Großbritannien, USA) durchgeführt wurde.

Insgesamt wurden 12546 Patienten eingeschlossen: männliche Patienten im Alter ab 55 Jahre und weibliche Patienten im Alter ab 60 Jahre mit einem mittleren kardiovaskulären Risikoprofil von 2-4 Risikofaktoren:

- hoher Cholesterinwert (> $200 \mathrm{mg} / \mathrm{dl}$ bei Männern/> $240 \mathrm{mg}$ bei Frauen) oder hohes LDL (> $130 \mathrm{mg} / \mathrm{dl}$ bei Männern/> $160 \mathrm{mg} / \mathrm{dl}$ bei Frauen),

- niedriges HDL (<40 mg/dl),

- Rauchen,

- arterielle Hypertonie (systolischer Blutdruck $>140 \mathrm{mmHg}$ ),

- antihypertensive Medikation,

- eine positive Familienanamnese hinsichtlich kardiovaskulärer Erkrankung.

Patienten wurden aus der Studie ausgeschlossen, falls sie bereits ein vaskuläres Ereignis wie beispielsweise einen Schlaganfall oder Herzinfarkt erlitten haben sowie an Herzrhythmusstörungen, Klappenschäden oder Herzinsuffizienz litten. Weitere Ausschlusskriterien beinhalteten ein hohes Risiko an gastrointestinalen oder intrazerebralen Blutungen als auch bereits überstandene gastrointestinale Blutungen oder Ulzerationen sowie Diabetes mellitus. Ein Großteil des Patientenkollektivs war bereits mit Antihypertensiva (64,8\%) oder mit Statinen (43\%) behandelt worden [18]. Das mediane Follow-up betrug 60 Monate.

Im 1:1 randomisierten Patientenkollektiv erhielt die ASSGruppe eine tägliche Gabe von ASS $100 \mathrm{mg}(\mathrm{n}=6270)$. Die Placebokontrollgruppe umfasste $n=6276$ Patienten. Tod durch ein kardiovaskuläres Ereignis, Myokardinfarkt, instabile Angina, Schlaganfall und transiente ischämische Attacke waren die primären Endpunkte der ARRIVE-Studie. 
Die Sicherheitsendpunkte bezogen sich auf hämorrhagische und andere schwerwiegende Ereignisse, wie beispielsweise Herzinfarkt, instabile Angina, transischämische Attacke oder Schlaganfall und wurden der Intention-to-treat-Population zugeordnet.

In der Intention-to-treat-Analyse trat der primäre Endpunkt bei 269 (4,29\%) Patienten in der Aspiringruppe gegenüber 281 (4,48\%) Patienten in der Placebogruppe auf (Hazard Ratio [HR] 0,96; 95\%-KI 0,81-1,13; p=0,6038). Gastrointestinale (meist leichte) Blutungsereignisse traten bei 61 (0,97\%) Patienten in der Aspiringruppe gegenüber $29(0,46 \%)$ in der Placebogruppe auf (HR 2,11; $95 \%-K I$ 1,36-3,28; $p=0,0007)$. Die Gesamtinzidenzrate schwerer unerwünschter Ereignisse war in beiden Behandlungsgruppen ähnlich ( $n=1266$ [20,19\%] gegenüber $n=1311$ [20,89\%] in der Placebogruppe). Die Gesamtinzidenz behandlungsbedingter unerwünschter Ereignisse war gering $(n=1050 \quad[16,75 \%]$ vs. $n=850$ [13,54\%] in der Placebogruppe; $p<0,0001)$. Es gab 321 dokumentierte Todesfälle in der Intention-to-treat-Gruppe $(n=160$ [2,55\%] vs. $n=161$ [2,57\%] von 6276 Patienten in der Placebogruppe).

Im Erreichen des primären Endpunkts gab es im 5-JahresFollow-up zwischen der ASS- und der Placebogruppe keine signifikanten Unterschiede (4,3\% unter Aspirin vs. 4,5\% unter Placebo), jedoch zeigte sich das Risiko für gastrointestinale Blutungen signifikant erhöht (mit 0,97\% bei der ASS-Gruppe doppelt so hoch wie in der Placebogruppe mit 0,46\%). Hinsichtlich Gesamtsterblichkeit und Rate fataler Blutungen gab es keine signifikanten Unterschiede zwischen den beiden Gruppen. Die Ereignisrate an primären Endpunkten war geringer, als von den Autoren erwartet wurde. Dies führen die Autoren der Studie auf die allgemeinen Präventionsstrategien wie die Statintherapie, physikalische Aktivitäten oder Diäten zurück [18].

Im Einklang mit der ASCEND-Studie konstatieren die Autoren, dass die ASS-Einnahme individuell mit dem Patienten entschieden werden muss hinsichtlich der kardiovaskulären Risikoreduktion und der Gefahr der gastrointestinalen Blutung.

\footnotetext{
Merke

Die ARRIVE-Studie hat gezeigt, dass ASS-Gabe nach 5 Jahren in der Intention-to-treat-Analyse (ITT) keinerlei Effekte auf die kardiovaskuläre Ereignisrate hat, das Risiko für gastrointestinale Blutungen jedoch mehr als verdoppelt.
}

\section{Ältere Patienten - ASPREE-Studie}

Das Ziel der dritten Studie, ASPREE („Effect of Aspirin on Disability-free Survival in the Healthy Elderly“), war es, zu klären, ob eine niedrige tägliche Dosis ASS die Zeit ohne körperliche Einschränkungen bei älteren Menschen verlängern könnte [20].

Zwischen 2010 und 2014 wurden in Australien und den Vereinigten Staaten 19114 Patienten in einem Alter von 70 Jahren oder älter (oder $\geq 65$ Jahre, wenn afroamerikanischer oder hispanischer Abstammung) rekrutiert. Die Ausschlusskriterien beinhalteten kardiovaskuläre Erkrankungen, Demenz oder physische Behinderungen in der Vorgeschichte.

Der primäre Endpunkt war Tod, beginnende Demenz oder eine physische Behinderung. Letztere wurde erreicht, wenn der Patient eine von den 6 grundlegenden Fertigkeiten zum täglichen Leben, wie Nahrungsaufnahme, Körperpflege oder der Toilettengang alleine, über ein halbes Jahr nicht mehr oder nur schwer ausüben könnte [20].

Die sekundären Endpunkte beinhalteten jegliche Form des Todes, einer größeren Blutung sowie einen hämorrhagischen Schlaganfall. Außerdem wurden tödliche und nicht tödliche kardiovaskuläre Erkrankungen, wie Herzinfarkt oder Schlaganfall, Krebserkrankungen, milde kognitive Einschränkung sowie Depressionen eingeschlossen [20].

Das eingeschlossene Patientenkollektiv erhielt randomisiert entweder täglich $100 \mathrm{mg}$ ASS (9525 Personen) oder ein Placebo (9589 Personen). Die Studie wurde nach einem medianen Follow-up von 4,7 Jahren bei einer erhöhten Blutungsneigung in der ASS-Gruppe frühzeitig beendet. Die Rate aller erreichten primären Endpunkte war in der ASS-Gruppe mit 21,5 Vorkommnissen nicht signifikant höher als in der Placebogruppe mit 21,2 Vorkommnissen pro 1000 Personenjahren (HR: 1,01; 95\%-KI $0,92-1,11 ; p=0,79)$. Vor allem die Anzahl an schwerwiegenden Blutungen war in der ASS-Gruppe im Vergleich zur Placebogruppe signifikant erhöht (3,8 vs. 2,8\%; HR: 1,38; 95\%-KI, 1,18-1,62; $\mathrm{p}<0,001)$ [21].

Der häufigste erreichte primäre Endpunkt war der Tod, welcher insgesamt ca. $50 \%$ der primären Endpunkte ausmacht (480/9525 in der ASS-Gruppe vs. 431/9589 Patienten in der Placebogruppe im Durchschnittsalter von 77,5 Jahren). Darauf folgt die Demenz mit 30\% der primär erreichten Endpunkte (274/9525 vs. 275/9589 Patienten im Durchschnittsalter von 77,7 Jahren), die persistierende körperliche Einschränkung wurde bei 375 Patienten (167/9525 vs. 208/9589) erreicht, welche $20 \%$ der primären Endpunkte umfasst [21].

Die Anzahl an Todesfällen jeglicher Ursache, welche in den sekundären Endpunkten der ASPREE-Studie definiert waren, war in der ASS-Gruppe mit 558 Patienten höher als in der Placebogruppe mit 494 Patienten. Dies bedeutet 12,7 vs. 11,1 Ereignisse pro 1000 Personenjahre (HR: 
1,14; 95\%-KI 1,01-1,29). Die Anzahl der 1052 (5,5\% des gesamten Patientenkollektivs) Todesfälle bemisst sich in der ASS-Gruppe auf 558 und in der Placebogruppe auf 494 Patienten. Dies bedeutet, dass das Risiko von Tod jeglicher Ursache in der ASS-Gruppe mit 12,7 vs. 11,1 Ereignissen pro 1000 Personenjahre (HR: 1,14; 95\%-KI 1,01$1,29)$ höher ist. Krebs war der Hauptgrund für die höhere Sterblichkeit in der ASS-Gruppe, welche 1,6 überzählige Todesfälle pro 1000 Personenjahre ausmachte. Krebsbedingte Todesfälle kamen bei 3,1\% der Teilnehmer in der ASS-Gruppe und bei 2,3\% der Teilnehmer in der Placebogruppe vor (HR: 1,31; 95\%-KI 1,10-1,56) [22].

Das Ergebnis der Studie ist, dass die prophylaktische Gabe von ASS, um bei älteren Patienten eine längere Zeit ohne körperliche Behinderungen zu erreichen, keinen Vorteil bringt. Es senkt zwar die Zahl an kardiovaskulären Ereignissen wie eines nicht tödlichen Herzinfarkts oder einer Hospitalisierung wegen einer Herzinsuffizienz (Rate an Ereignissen pro 1000 Personenjahre, ASS 10,7 vs. 11,3, HR: 1,38\%; 95\%-KI 1,18-1,62; p<0,001) [18], allerdings steigt die Zahl an schwerwiegenden Blutungen wie symptomatischen intra- oder extrakraniellen Blutungen oder hämorrhagischen Schlaganfällen in der ASS-Gruppe an (Rate an Ereignissen pro 1000 Personenjahre, ASS $3,8 \%$ vs. $2,8 \%$, HR: 1,38 ; $95 \%-K I$ 1,18-1,62; $p<0,001$ ) [21].

\footnotetext{
Merke Tod, den Beginn einer Demenz oder einer physischen Behinderung) durch eine primärprophylaktische ASS-Einnahme, vielmehr steigt das Blutungsrisiko signifikant an.
}

\section{Körpergewicht und Dosierung - „Effects of aspirin on risks of vascular events and cancer according to bodyweight and dose"-Studie}

In Deutschland ist ASS in Dosierungen von $100 \mathrm{mg} / \mathrm{d}$ und $300 \mathrm{mg} / \mathrm{d}$ zur Therapie und Prophylaxe kardiovaskulärer Erkrankungen zugelassen. Im Gegensatz dazu ist ASS in den USA in den Dosierungen von $81 \mathrm{mg} / \mathrm{d}$ (low-dose) und 325 mg/d (regular-dose) erhältlich.

Im Falle eines akuten Myokardinfarkts sollte laut der Leitlinie von 2017 der European Society of Cardiology eine initiale Dosis von 150-300 mg p.o. bzw. von 75-250 mg i.v., wenn eine orale Einnahme nicht möglich ist, verabreicht werden [23].

Die Studie „Effects of aspirin on risks of vascular events and cancer according to bodyweight and dose" hat sich mit der Fragestellung beschäftigt, ob die ASS-Dosis in der kardiovaskulären Prävention an das Körpergewicht der Patienten angepasst werden muss [24].
Das Patientenkollektiv für diese retrospektive Analyse wurde aus mehreren Studien des Antithrombotic Treatment Trialists' (ATT) Collaboration, welche ASS in der Primär- als auch Sekundärprävention untersucht hatten, ausgewählt. Gewicht, Alter, Geschlecht, vaskuläre Risikofaktoren, wie beispielsweise hohes LDL, Rauchen und der Diabetesstatus, wurden erfasst.

Einerseits wurde zunächst unterschieden, ob ASS initial zur Primär- oder Sekundärprävention eingesetzt wurde, andererseits wie hoch die Initialdosis war $(100 \mathrm{mg}$ oder $>300 \mathrm{mg}$ ). Anschließend wurden diese verschiedenen Gruppen in Alter, Geschlecht, Größe und Gewicht weiter differenziert. Schließlich wurde im letzten Schritt jede Patientengruppe in 2 Bereiche unterteilt:

- <70 kg Körpergewicht,

- $\geq 70$ kg Körpergewicht.

Innerhalb dieser beiden Patientengruppen gab es noch jeweils 2 weitere Subgruppen:

- In dem Patientenkollektiv < 70 kg Körpergewicht wurde zwischen $<50 \mathrm{~kg}$ sowie $50-69 \mathrm{~kg}$ Körpergewicht unterschieden.

- In dem Patientenkollektiv $\geq 70$ kg Körpergewicht wurde zwischen $\geq 70-89 \mathrm{~kg}$ und über $\geq 90 \mathrm{~kg}$ Körpergewicht differenziert.

Für jedes Ergebnis wurde das Risikoverhältnis (HR) zwischen der ASS-Gruppe und Placebogruppe ausgerechnet. Des Weiteren wurden mithilfe des Cox-Regressionsmodells der Effekt von ASS bei Patienten unter $<70 \mathrm{~kg}$ oder $\geq 70 \mathrm{~kg}$ Körpergewicht stratifiziert. Außerdem wurden gewichtsstratifizierte Analysen im Hinblick auf den Effekt von ASS auf tödliche Ereignisse, Schlaganfall und Myokardinfarkt gemacht. Zusätzlich wurde der Effekt von ASS hinsichtlich des Risikos aller kardiovaskulären Ereignisse sowie Todes jeglicher Ursache untersucht.

In dem Patientenkollektiv mit weniger als 70 kg Körpergewicht und einer täglichen niedrigdosierten ASS-Dosis (75-100 mg) wurden 2 weitere Subgruppen verglichen:

- Patienten < 50 kg Körpergewicht,

- Patienten mit einem Körpergewicht von 50-69 kg Körpergewicht.

Bei letzterer Patientengruppe wurden im Vergleich zur Kontrollgruppe weniger kardiovaskuläre Events festgestellt (383 Ereignisse bei 15155 Patienten mit ASS vs. 504 Ereignisse bei 15145 Patienten mit Placebo; HR: 0,75; 95\%-KI 0,75-0,885; $\mathrm{p}<0,0001)$. ASS hatte allerdings keinen primärprophylaktischen Nutzen mehr bei der Patientengruppe mit einem Körpergewicht von unter 50 kg (HR: 1,25; 95\%-KI 0,74-2,09; p=0,40). Der gewichtsabhängige Effekt der niedrigdosierten ASS-Gabe wurde in allen Patientenkollektiven unter 70 kg Körpergewicht festgestellt. Mit ansteigendem Körpergewicht nimmt die primärpräventive Wirkung der niedrigdosier- 
ten ASS-Gabe (75-100 mg) ab. Bei Patienten mit einem Körpergewicht über $70 \mathrm{~kg}$ wurde im Vergleich zur Kontrollgruppe unter einer niedrigdosierten ASS-Therapie ein Anstieg der Todesfälle bei einem ersten kardiovaskulären Ereignis erfasst (OR: 1,33; 95\%-KI 1,08-1,64; $\mathrm{p}=0,0082$ ). Insbesondere wurde dies bei Myokardinfarkten beobachtet (OR: 1,73; 95\%-KI 1,20-2,49; $p=0,0035)$. Unabhängig vom Körpergewicht stieg unter einer Low-Dose-ASS-Therapie im Rahmen der Primärprävention der Anteil an erstmalig auftretenden und tödlichen vaskulären Ereignissen bei Patienten ab der 7. Lebensdekade an (HR: 1,45; 95\%-KI 1,01-2,10; $p=0,04$ ) [24].

In der Sekundärprophylaxe mit ASS (50 mg/d) nach einem Schlaganfall zeigten Patienten unter 70 kg Körpergewicht gegenüber dem Kontrollkollektiv eine reduzierte Rate an kardiovaskulären Ereignissen (HR: 0,74; 95\%-KI 0,63$0,87 ; p=0,0003)$. Die niedrigdosierte ASS-Gabe von 75$100 \mathrm{mg}$ hatte im Vergleich zur Kontrollgruppe vor allem bei Frauen unter 70 kg Körpergewicht im Rahmen der Sekundärprävention eine senkende Wirkung auf das Risiko, einen plötzlichen Tod oder ein kardiovaskuläres Ereignis zu erleiden (HR 0,68; 95\%-KI 0,56-0,83; p=0,0001). Bei Frauen über 70 kg Körpergewicht wurde das Risiko, verglichen mit der Kontrollgruppe unter der niedrigdosierten ASS-Einnahme als Sekundärprophylaxe, nicht signifikant gesenkt (HR: 1,02; 95\%-KI 0,77-1,35; p =0,90) [24].

Ab einem Körpergewicht über $70 \mathrm{~kg}$ reduziert eine hohe ASS-Dosis (300-325 mg/d) verglichen zur Kontrollgruppe das Risiko, ein kardiovaskuläres Ereignis zu erleiden, welches als Herzinfarkt, Schlaganfall oder Tod definiert ist (HR 0,79; 95\%-KI 0,70-0,90; $\mathrm{p}=0,005$ ). Patienten mit einem Körpergewicht von $>90 \mathrm{~kg}$ benötigen über $500 \mathrm{mg} / \mathrm{d}$ ASS, um eine effektive Risikoreduktion (HR: 0,45; 95\%-KI 0,26-0,79; $\mathrm{p}=0,005)$ zu erzielen [24].

Diese Effekte zeigen, dass das Körpergewicht mit der ASSDosis in Wechselwirkung steht. Durch eine genauere Anpassung kann möglicherweise die Effektivität des Thrombozytenaggregationshemmers verbessert werden. Des Weiteren schließen die Autoren aus der Studie, dass mehr als $80 \%$ der Männer und mehr als $50 \%$ der Frauen aufgrund eines höheren Körpergewichts von 70 kg hinsichtlich der eingenommenen ASS-Dosis unterdosiert sind.

Dies könnte eine Erklärung für die Ergebnisse der ARRIVEund ASCEND-Studien sein, in welchen publiziert wurde, dass eine Therapie mit ASS 100 mg/d in der Primärprävention von kardiovaskulären Ereignissen keine durchschlagenden Erfolge erzielt hat. Weitere Studien zu einer gewichtsbasierten ASS-Dosis müssen dieses Phänomen bestätigen, bevor eine gewichtsadaptierte Dosierung empfohlen werden kann.
Merke

Die wirksame ASS-Dosis scheint gewichtsabhängig zu sein.

\section{Fazit}

Die Daten großer randomisierter Studien wie ASPREE, ARRIVE und ASCEND sprechen gegen eine primärprophylaktische Therapie mit ASS. Diese Schlussfolgerung wird durch Metaanalysen unterstützt, die keinen Benefit einer primärprophylaktischen ASS-Medikation auf die Gesamtmortalität aufzeigen. Das Risiko schwerer Blutungen überwiegt den geringen Benefit hinsichtlich niedrigerem Auftreten nicht tödlicher Myokardinfarkte, TIAs und ischämischen Schlaganfällen [25].

\section{ASS in der Sekundärprävention}

In der Sekundärprävention ist der Nutzen ASS durch viele Studien gut belegt - die niedrigdosierte ASS-Gabe (75100 mg/d) hat sich bei Patienten mit einem hohen kardiovaskulären Risiko, nach einem Myokardinfarkt oder einem ischämischen Schlaganfall, seit Jahren bewährt und ist in den Leitlinien verankert [1]. Laut den ESC/ ESAC-Leitlinien sollte auch nach einer perkutanen koronaren Intervention eine dauerhafte Gerinnungshemmung mit ASS (75-100 mg/d) erfolgen [26].

Bei Patienten, die einen Myokardinfarkt (MI) oder ischämischem Schlaganfall erlitten haben, besteht ein sehr hohes Risiko, ein weiteres CVD-Ereignis zu erleiden. Eine Aspirin-Therapie kann dazu dienen, nachfolgende CVDEreignisse zu verhindern [27].

Die Rolle von Aspirin bei der Verringerung der Letalität durch CDV und der Wiederholung von Ereignissen nach einem akuten MI wurde erstmals 1988 in der zweiten Studie der International Study of Infarct Survival (ISIS-2) nachgewiesen [28]. In dieser Studie wurden $17187 \mathrm{~Pa}$ tienten aus 417 Krankenhäusern innerhalb von 24 Stunden nach Ausbruch des Verdachts auf einen akuten MI rekrutiert und in folgende Gruppen randomisiert:

- 1-stündige intravenöse Infusion von 1,5 MU Streptokinase,

- 1 Monat lang $160 \mathrm{mg} / \mathrm{d}$ p.o. Aspirin (magensaftresistent beschichtet),

- beide Behandlungen,

- keine von beiden.

Die Einnahme von Aspirin führte zu einer signifikanten Verringerung der nicht tödlichen Re-Infarkte, Schlaganfälle, der 5-wöchigen vaskulären Letalität und der Gesamtletalität. Obwohl andere kleinere Studien ähnliche Vorteile für Patienten mit einer Vorgeschichte von früherem MI zeigten, war die ISIS-2-Studie die erste, die einen Nachweis für eine direkte Wirkung von Aspirin auf den Verlauf eines akuten Ml erbrachte. Sie zeigte, dass die Gabe von niedrigdosiertem Aspirin für die Dauer eines 
Monats, unmittelbar begonnen nach einem MI bei 1000 Patienten, 25 Todesfälle und 10-15 nicht tödliche Infarkte und Schlaganfälle verhindern würde. Ein zusätzlicher Nutzen für die Letalität wurde bei längerer Dauer der Aspirintherapie beobachtet $[27,28]$.

Seither haben auch einige andere Gruppen ähnliche Ergebnisse zum Einsatz von Aspirin in der Sekundärprävention von CVD-Ereignissen publiziert.

In der Metaanalyse der Antithrombotic Treatment Trialists' (ATT) Collaboration aus dem Jahr 2009 wurden z. B. retrospektiv der Nutzen und die Risiken einer ASS-Therapie (zwischen 50 und 1500 mg/d) in der Primär- und Sekundärprävention evaluiert. Der Endpunkt waren schwerwiegende vaskuläre Ereignisse (Herzinfarkt, Schlaganfall oder vaskulärer Tod) [29].

In 16 Studien zur Langzeitanwendung von Aspirin mit Dosen zur Sekundärprävention von CVD-Ereignissen, darunter über 17000 Probanden und 3306 schwere vaskuläre Ereignisse, zeigte ASS eine signifikante Reduktion der schwerwiegenden vaskulären Ereignisse (Herzinfarkt, Schlaganfall, Tod) (6,7 vs. 8,2\% pro Jahr; $p<0,0001)$. Sowohl die Anzahl schwerer vaskulärer Ereignisse (995 [4,3\%] vs. 1214 [5,3\%] Events pro Jahr; 95\%-KI 0,80; $\mathrm{p}<0,00001)$ als auch bei schweren vaskulären Ereignissen (1505 [6,69\%] vs. 1801 [8,19\%]; 95\%-KI 0,81; $\mathrm{p}<0,00001)$ konnte durch ASS signifikant gegenüber der Placebogruppe gesenkt werden. Nicht tödliche Herzinfarkte (357 vs. 505 Events; 95\%-KI 0,69) sowie Patienten mit einer KHK (614 vs. 696 Events; 95\%-KI 0,87) profitierten durch eine Sekundärprävention mit ASS. Niedrigdosierungen $(75-100 \mathrm{mg} / \mathrm{d})$ erwiesen sich als ebenso wirksam wie höhere Dosen [29].

Die Anzahl an ischämischen Schlaganfällen konnte in der ASS-Gruppe ebenfalls gesenkt werden (140 [0,61\%] vs. 176 [0,77\%]; 95\%-KI 0,78; $p=0,04)$. Das Risiko, eine Hirnblutung (36 vs. 19 Events; 95\%-KI 1,39; $p=0,01$ ) zu erleiden, stieg an, jedoch war dieser Anstieg nicht signifikant [29].

Patienten mit einer peripheren arteriellen Verschlusskrankheit wurden in der randomisierten doppelblinden Studie CLIPS („Critical Leg Ischaemia Prevention Study“) hinsichtlich des protektiven Effekts von ASS untersucht. Das Ziel der Studie war es, die prophylaktische Wirksamkeit von Aspirin und einer hochdosierten antioxidativen Vitaminkombination bei Patienten mit peripherer arterieller Verschlusskrankheit im Hinblick auf die Verringerung des Risikos eines ersten vaskulären Ereignisses (Myokardinfarkt, Schlaganfall, vaskulärer Tod) und einer kritischen Extremitätenischämie zu untersuchen.

Insgesamt erlitten 7 von 185 (4\%) Personen der ASSGruppe im Vergleich zu 20 von 181 (11\%) der Placebo- gruppe ein schwerwiegendes vaskuläres Ereignis (2 vs. 11 Herzinfarkt; 4 vs. 7 Schlaganfälle; 1 vs. 2 Lungenembolien). Dies bedeutet eine Risikoreduktion von 64\% (HR: 0,35; 95\%-KI 0,15-0,82; p=0,016). Des Weiteren war die Anzahl an kritischen Extremitätenischämien durch die antithrombozytäre Therapie geringer (5/185 vs. 8/181) [30]. Folglich sollte im Rahmen einer symptomatischen peripheren arteriellen Verschlusskrankheit eine antithrombozytäre Therapie in der Sekundärprävention erfolgen [31].

\section{ASS in Kombination mit anderen Thrombozytenaggregationshemmern}

Neben ASS zählen auch P2Y12-Antagonisten zu der Gruppe der Thrombozytenaggregationshemmer. Diese blockieren den ADP-Rezeptor P2Y12 auf der Oberfläche der Thrombozyten, mit der Folge, dass ADP nicht binden kann und die Thrombozytenaggregation über die Glykoprotein-IIb/lla-Aktivierung inhibiert wird [26].

In der CAPRIE-Studie („A randomised, blinded, trial of clopidogrel versus aspirin in patients at risk of ischaemic events") wurde die antithrombotische Wirksamkeit von Clopidogrel $(75 \mathrm{mg} / \mathrm{d})$, einem P2Y12-Antagonisten der 2. Generation, mit ASS (325 mg/d) hinsichtlich der Risikoreduktion eines kombinierten Endpunkts bestehend aus Myokardinfarkt, ischämischem Schlaganfall oder vaskulärem Tod, über einen Zeitraum von 1-3 Jahren verglichen. In der Studie reduzierte Clopidogrel im Vergleich zu ASS den kombinierten Endpunkt, bestehend aus kardiovaskulärem Tod, Herzinfarkt und Schlaganfall, um relative 8,7\% ohne Unterschied in Gesamtblutungsereignissen und bei intrakraniellen Blutungen. Die individuellen Endpunkte und die Gesamtsterblichkeit waren zwischen ASS und Clopidogrel nicht signifikant unterschiedlich. Die Rate gastrointestinaler Blutungen war unter ASS gegenüber Clopidogrel (2,65 vs. 2,0\%) erhöht. In der Subgruppe der Patienten mit KHK (Zustand nach Herzinfarkt) war ASS so effektiv und sicher wie Clopidogrel, sodass Clopidogrel bei KHK nur bei ASS-Unverträglichkeit gegeben werden sollte [32].

In der TiCAB-Studie wurde die Wirkung von ASS mit jener von Ticagrelor bei Patienten nach koronarer Bypassoperation verglichen [33]. Nach einer Follow-up-Periode von 12 Monaten ergab sich kein signifikanter Unterschied in den ischämischen und in den Blutungsereignissen zwischen beiden Gruppen. Die Studie wurde jedoch vorzeitig wegen Finanzierungsproblemen gestoppt, nachdem erst die Hälfte der ursprünglich geplanten Studienteilnehmer rekrutiert worden war. Weitere Studien beschäftigten sich mit dem Vergleich der dualen Thrombozytenhemmung im Vergleich zur Monotherapie mit ASS. Die CUREsowie die COMMIT-Studien fanden dabei bei Patienten mit akuten koronaren Syndromen einen Vorteil in der dualen Thrombozytenhemmung mit ASS und einem P2Y12-Rezeptor-Inhibitor gegenüber der Monotherapie 
mit ASS [34, 35]. Im Gegensatz dazu war dies in der CHARISMA-Studie [36] bei Patienten mit chronischer KHK nicht der Fall. Nur bei Patienten, welche in der Vorgeschichte einen Herzinfarkt erlitten hatten, konnten Vorteile in der dualen Thrombozytenhemmung (Clopidogrel + ASS) gegenüber einer Behandlung mit ASS alleine gefunden werden [37]. Auch in der PEGASUS-Studie [38] wurde ein Vorteil der über den Zeitraum von 12 Monaten nach Herzinfarkt hinausgehenden dualen Therapie (Ticagrelor + ASS) gegenüber der ASS-Monotherapie festgestellt.

In der GLOBAL-LEADERS-Studie [39] wurde eine duale Thrombozytenhemmung mit Ticagrelor über 4 Wochen, gefolgt von einer Monotherapie mit Ticagrelor über weitere 23 Monate, gegenüber einer Standardtherapie mit ASS und Clopidogrel über 12 Monate nach Herzinfarkt sowie ASS und Clopidogrel über 12 Monate nach elektiver $\mathrm{PCl}$ untersucht. Das Resultat: Ticagrelor in Kombination mit Aspirin für 1 Monat, gefolgt von Ticagrelor allein für 23 Monate, war einer 12-monatigen dualen StandardThrombozytenaggregationshemmer-Therapie, gefolgt von 12 Monaten Aspirin allein, nicht überlegen in der Senkung der Gesamtletalität oder Prävention eines erneuten Myokardinfarkts 2 Jahre nach perkutaner Koronarintervention.

In der TWILIGHT-Studie [40] wurde eine 12-monatige Monotherapie mit Ticagrelor im Vergleich zu einer dualen Thrombozytenhemmung mit ASS und Ticagrelor nach einer 3-monatigen dualen Thrombozytenhemmung nach Stentimplantation untersucht. Im Gegensatz zur GLOBALLEADERS-Studie wurde hier gefunden, dass bei Hochrisikopatienten, die eine $\mathrm{PCl}$ bekamen und eine 3-monatige duale Thrombozytenaggregationshemmer-Therapie durchliefen, die Ticagrelor-Monotherapie mit einer geringeren Inzidenz klinisch relevanter Blutungen verbunden war als Ticagrelor plus Aspirin, ohne dass ein höheres Risiko für Tod, Myokardinfarkt oder Schlaganfall bestand.

In der COMPASS-Studie („Cardiovascular Outcome for people using anticoagulation strategies“) [41] wurde die Inzidenzrate für die Auswirkung einer Therapie mit ASS $(100 \mathrm{mg} / \mathrm{d})$ mit zusätzlich dem direkten Faktor-Xa-Inhibitor Rivaroxaban $(2 \times 2,5 \mathrm{mg} / \mathrm{d})$ gegenüber einer Monotherapie mit ASS $(100 \mathrm{mg} / \mathrm{d})$ bei kardiovaskulären Hochrisikopatienten verglichen. Die Patienten mussten entweder eine stabile koronare Herzerkrankung oder eine periphere arterielle Verschlusskrankheit aufweisen. Der kombinierte Endpunkt bestand aus einem kardiovaskulär bedingten Tod sowie einem Erstauftritt eines Myokardinfarkts oder ischämischen Schlaganfalls. Gefährliche Blutungen, Tod oder blutungsbedingte Hospitalisierung waren die primären Sicherheitspunkte. In der Kombinationstherapie wurde der primäre Endpunkt relativ um 24\% zur Monotherapie mit ASS reduziert (HR: 0,80; $95 \%-K I 0,70-0,91 ; p=0,0005)$. Innerhalb der Studien- dauer von 23 Monaten wurde der primäre Endpunkt bei der Rivaroxaban/ASS-Gruppe bei 379 und bei der ASSGruppe bei 479 Patienten erreicht (4,1 vs. 5,4\%). Allerdings traten Blutungen signifikant häufiger unter der Kombinationstherapie (3,1\%) im Vergleich zur Monotherapie mit ASS (1,9\%) auf (HR: 1,70; $<<0,001)$ [41].

Eine tägliche niedrigdosierte ASS-Gabe im Rahmen der Sekundärprävention reduziert bei Hochrisikopatienten also das Risiko, einen erneuten Herzinfarkt, Schlaganfall oder ein anderes vaskuläres Ereignis zu erleiden. Die Häufigkeit von hämorrhagischen Schlaganfällen stieg von 1 auf 2 Ereignisse pro 10000 Patienten. Schließlich wiegt der Nutzen einer niedrigdosierten ASS-Gabe von 75$100 \mathrm{mg}$ bei Hochrisikopatienten das Risiko, eine Blutung zu erleiden, auf.

Merke
ASS $100 \mathrm{mg} / \mathrm{d}$ ist bei kardiovaskulären Hochrisiko-
patienten im Rahmen der Sekundärprävention
indiziert und kann als Standard der Thrombozyten-
hemmenden Therapie bezeichnet werden.

\section{Zusammenfassung}

Zusammenfassend beschäftigten sich die vorgestellten Studien mit der Frage, ob eine Primärprophylaxe mit ASS hinsichtlich eines kardiovaskulären Nutzens bei verschiedenen Risikoprofilen sinnvoll ist. Eingeschlossene Risikoprofile waren Patienten mit einem niedrigen bis mittleren kardiovaskulären Risiko, Diabetiker und Patienten $>65$ Jahre.

Die Ergebnisse zeigen, dass Patienten mit einem niedrigen oder niedrig bis mäßigen Risikoprofil für eine HerzKreislauf-Erkrankung ebenso wie Diabetiker nicht ausreichend stark von einer Primärprävention mit ASS profitieren, sodass hierbei die Nebenwirkungen überwiegen. Eine Blutung als Komplikationsmöglichkeit zu erleiden, ist die größte Gefahr und steigt stark bei einer täglichen Einnahme von ASS an. Dementsprechend wird eine primäre Prävention mit ASS bei diesen Patientengruppen nicht beziehungsweise nur unter individueller Beratung und Abschätzung der Risiken empfohlen ( $\bullet$ Abb. 2).

Patienten > 70 Jahren mit einer hohen kardiovaskulären Gefährdung würden hinsichtlich nicht tödlicher Herzinfarkte und Hospitalisierung von der präventiven ASSEinnahme profitieren, jedoch ging damit auch ein erhöhtes Blutungsrisiko bei dieser Patientengruppe einher [18]. Dies basiert auf dem zunehmenden Blutungsrisiko im Alter sowie den Nebenwirkungen der täglichen ASS-Einnahme [20]. Generell sollten Patienten ab der 7. Lebensdekade, vor allem jene mit einer hohen Sturzneigung, ASS nicht primärpräventiv erhalten. 


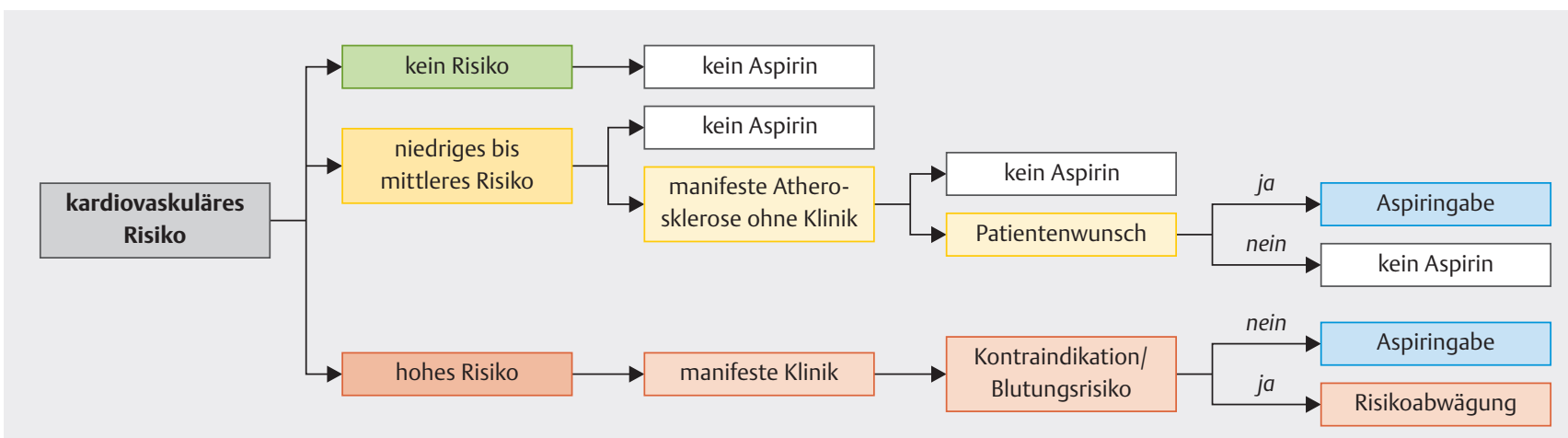

- Abb. 2 Diagramm zur Empfehlung einer täglichen ASS-Gabe (100 mg/d) im Rahmen der Primärprävention.

Patienten nach einem kardiovaskulären Event sollten im Rahmen einer Sekundärprävention eine tägliche niedrigdosierte ASS-Gabe $(100 \mathrm{mg} / \mathrm{d})$ erhalten. Hierbei hat sich in Studien gezeigt, dass durch eine ASS-Einnahme die Anzahl an schwerwiegenden kardiovaskulären Ereignissen deutlich geringer war im Vergleich zu der Placebogruppe.

In der Sekundärprävention überwiegt der Vorteil der täglichen ASS-Einnahme das Blutungsrisiko als Nebenwirkung. Falls eine Unverträglichkeit vorhanden sein sollte, muss auf einen P2Y12-Inhibitor umgestellt werden [1].

Ein Großteil des Patientenkollektivs wurde bereits im Vorfeld mit Antihypertensiva oder Statinen behandelt. Hinzu kommen allgemeine Präventionsstrategien wie physikalische Aktivitäten oder Diäten, welche in der Allgemeinbevölkerung Einzug gefunden haben. Dies scheint nach der Meinung der Autoren der Studien bereits eine gute Basis zur Verhinderung eines kardiovaskulären Ereignisses zu sein, sodass eine mögliche On-Top-Prophylaxe mit einer täglichen ASS-Einnahme dadurch keinen signifikanten Unterschied macht.

Die Studie „Effects of aspirin on risks of vascular events and cancer according to bodyweight and dose“ [24] zeigt interessante Ansätze zur optimalen Dosierung von ASS. Möglicherweise sind laut der Studie die meisten Patienten mit ASS unterdosiert und dementsprechend zeigt sich kein Vorteil im Rahmen einer Primärprävention. Weitere Studien müssen diesen Effekt untersuchen bevor relevante Rückschlüsse für die tägliche Praxis daraus gezogen werden können.

Den negativen Daten aus o.g. großen, randomisierten Studien und Metaanalysen folgend, sprechen sich die Leitlinien sowohl der europäischen als auch der US-amerikanischen kardiologischen Gesellschaft gegen den Einsatz von ASS in der Primärprävention aus (Klasse III oder Ilb Empfehlungen) ( $\vee$ Tab. 2 bis $\triangleright$ Tab. 4). 
- Tab. 2 Aktuelle Leitlinienempfehlungen für den Gebrauch von ASS in der Primärprävention von kardiovaskulären Erkrankungen.

\section{Organisation}

2016 European Guidelines on Cardiovascular Disease Prevention in Clinical Practice [42]

American College of Chest Physicians Guideline on Primary and Secondary Prevention of Cardiovascular Disease 2012 [43]

American Diabetes Association Standards of Medical Care - 2019 [44]

2019 American College of Cardiology/American Heart Association Guideline for Primary Prevention of Cardiovascular Disease [45]

\section{Empfehlung}

keine antithrombotische Behandlung von Diabetikern oder Menschen ohne kardiovaskuläre Erkrankung

niedrigdosiertes ASS (75-100 mg/d) für Patienten > 50 Jahre ohne symptomatische kardiovaskuläre Erkrankung

niedrigdosiertes ASS (75-162 mg/d) für Diabetiker $>50$ Jahre mit mindestens einem weiteren kardiovaskulären Risikofaktor und niedrigem Blutungsrisiko nach individueller Nutzen-Risiko-Abwägung

mögliche Gabe von niedrigdosiertem ASS (75-100 mg/d) bei Patienten zwischen 40-70 Jahren mit einem erhöhten Risiko für atherosklerotische kardiovaskuläre Erkrankungen ohne erhöhtes Blutungsrisiko

keine Gabe bei Patienten > 70 Jahre

keine Gabe bei Patienten mit erhöhtem Blutungsrisiko

\section{Empfehlungs- grad}

III

Evidenzklasse

A/B

II

B

III

C

A

IIb $\quad$ A

III

III

> Tab. 3 Definition des Empfehlungsgrades/Class of recommendation. Modifiziert nach Centre of Evidence-Based Medicine.

\begin{tabular}{|l|l|}
\hline Grad der Empfehlung & Grundlage \\
\hline $1 \mathrm{a}$ & Evidenz aufgrund von Metaanalysen mehrerer randomisierter, kontrollierter Studien \\
\hline $1 \mathrm{~b}$ & Evidenz aufgrund mindestens einer randomisierten, kontrollierten Studie \\
\hline $2 \mathrm{a}$ & Evidenz aufgrund mindestens einer gut angelegten, nicht randomisierten oder kontrollierten Studie \\
\hline $2 \mathrm{~b}$ & Evidenz aufgrund mindestens einer gut angelegten quasi-experimentellen Studie \\
\hline 3 & Evidenz aufgrund gut angelegter, nicht experimenteller, deskriptiver Studien \\
\hline 4 & Evidenz aufgrund von Fallberichten \\
\hline 5 & Evidenz aufgrund von Berichten der Expertenausschüsse oder Expertenmeinung anerkannter Autoritäten \\
\hline
\end{tabular}

- Tab. 4 Definition der Evidenzklassen/Level of evidence. Modifiziert nach Centre of Evidence-Based Medicine.

\begin{tabular}{|l|}
\hline Grad der Empfehlung \\
\hline A \\
\hline B \\
\hline C \\
\hline D \\
\hline
\end{tabular}

\section{Definition}

Erkenntnisse basierend auf gesicherten Level-1-Studien

Erkenntnisse basierend auf gesicherten Level-2- oder -3-Studien oder Extrapolation von Level-1-Studien

Erkenntnisse basierend auf Level-4-Studien oder Extrapolation von Level-2- oder -3-Studien

Erkenntnisse basierend auf Level-4-Studien oder nicht gesicherter Studien aller Evidenzklassen

\section{KERNAUSSAGEN}

- ASS wirkt als Thrombozytenaggregationshemmer.

- Große randomisierte Studien wie ASCEND, ARRIVE und ASPREE haben die Wirksamkeit und Sicherheit von ASS in der Primärprävention untersucht.

- Die Risiken, Blutungen zu erleiden, überwiegen den potenziellen Nutzen von ASS in der Primärprävention.

- Nur nach sorgfältiger Nutzen-Risiko-Abwägung kommen einzelne Patienten für eine primärprophylaktische ASS-The- rapie infrage. Kriterien hierfür sind: Diabetes mellitus, 10-jahres-Risiko für kardiovaskuläre Ereignisse $>10 \%$, keine Statintherapie sowie niedriges Risiko für gastrointestinale Blutungen.

- ASS 100 mg/d ist bei kardiovaskulären Hochrisikopatienten im Rahmen der Sekundärprävention indiziert und kann als Standard der Thrombozyten-hemmenden Therapie bezeichnet werden. 
Erklärung zu finanziellen Interessen

Forschungsförderung erhalten: nein; Honorar/geldwerten Vorteil für Referententätigkeit erhalten: nein; Bezahlter Berater/interner Schulungsreferent/Gehaltsempfänger: nein; Patent/Geschäftsanteile/Aktien (Autor/Partner, Ehepartner, Kinder) an im Bereich der Medizin aktiven Firma: nein; Patent/ Geschäftsanteile/Aktien (Autor/Partner, Ehepartner, Kinder) an zu Sponsoren dieser Fortbildung bzw. durch die Fortbildung in ihren Geschäftsinteressen berührten Firma: nein.

Erklärung zu nichtfinanziellen Interessen

Die Autorinnen/Autoren geben an, dass kein Interessenkonflikt besteht.

\section{Autorinnen/Autoren}

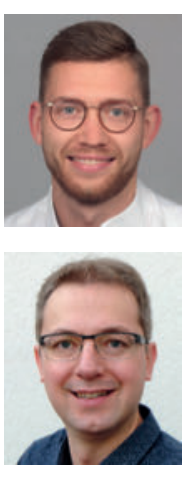

\section{Alexander Hach}

Jahrgang 1992. Student der Humanmedizin an der Universität zu Lübeck.

\section{Reinhard Sauter}

Dr. med. Jahrgang 1983. Facharzt in der Medizinische Klinik II/Kardiologie, Angiologie, Intensivmedizin, UKSH, Campus Lübeck.

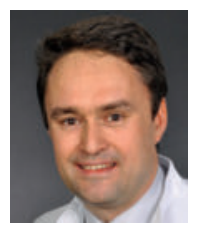

\section{Harald Langer}

Prof. Dr. med. Jahrgang 1976. Sektionsleiter, Sektion für präventive und translationale Kardiologie, Medizinische Klinik I//Kardiologie, Angiologie, Intensivmedizin, UKSH, Campus Lübeck.

\section{Korrespondenzadresse}

\author{
Dr. med. Reinhard Sauter \\ Medizinische Klinik II \\ (Kardiologie, Angiologie, Intensivmedizin) \\ Universitäres Herzzentrum Lübeck \\ Ratzeburger Allee 160 \\ 23538 Lübeck \\ Deutschland \\ reinhardjoerg.sauter@uksh.de
}

\section{Wissenschaftlich verantwortlich}

\section{gemäß Zertifizierungsbestimmungen}

Wissenschaftlich verantwortlich gemäß Zertifizierungsbestimmungen für diesen Beitrag ist Dr. med. Reinhard Sauter, Lübeck.

\section{Literatur}

[1] Patrono C, García Rodríguez LA, Landolfi R et al. Low-Dose Aspirin for the Prevention of Atherothrombosis. N Engl J Med 2005; 353: 2373-2383

[2] Walker J, Hutchison P, Ge J et al. Aspirin: 120 years of innovation. A report from the 2017 Scientific Conference of the International Aspirin Foundation, 14 September 2017, Charité, Berlin. Ecancermedicalscience 2018; 12: 813

[3] Patrignani P, Patrono C. Cyclooxygenase inhibitors: From pharmacology to clinical read-outs. Biochim Biophys Acta 2015; 4: 422-432

[4] Patrono C. Aspirin as an antiplatelet drug. N Engl J Med 1994; 330: 1287-1294

[5] Patrono C. The Multifaceted Clinical Readouts of Platelet Inhibition by Low-Dose Aspirin. J Am Coll Cardiol 2015; 66: 74-85

[6] FitzGerald GA, Brash AR, Oates JA et al. Endogenous biosynthesis of prostacyclin and thromboxane and platelet function during chronic administration of aspirin in man. J Clin Invest 1983; 71: 676-688

[7] Patrignani P, Filabozzi P, Patrono C. Selective Cumulative Inhibition of Platelet Thromboxane Production by Low-dose Aspirin in Healthy Subjects. J Clin Invest 1982; 69: 1366-1372

[8] Undas A, Brummel-Ziedins K, Mann KG. Why does aspirin decrease the risk of venous thromboembolism? On old and novel antithrombotic effects of acetyl salicylic acid. J Thromb Haemost 2014; 12: 1776-1787

[9] Thun M], Henley S], Patrono C. Nonsteroidal anti-inflammatory drugs as anticancer agents: mechanistic, pharmacologic, and clinical issues. J Natl Cancer Inst 2002; 94: 252-266

[10] Auriel E, Regev K, Korczyn AD. Nonsteroidal anti-inflammatory drugs exposure and the central nervous system. Handb Clin Neurol 2014; 119: 577-584

[11] Bloom BS. Direct medical costs of disease and gastrointestinal side effects during treatment for arthritis. Am J Med 1988; 84: 20-24

[12] Varghese M, Lockey RF. Aspirin-exacerbated asthma. Allergy Asthma Clin Immunol 2008; 4: 75-83

[13] Starko KM, Ray CG, Dominguez LG et al. Reye's syndrome and salicylate use. Pediatrics 1980; 66: 859-864

[14] Pirmohamed M, James S, Meakin S et al. Adverse drug reactions as cause of admission to hospital: prospective analysis of 18820 patients. BMJ 2004; 329: 15-19

[15] Crofford LJ. COX-1 and COX-2 tissue expression: implications and predictions. J Rheumatol Suppl 1997; 49: 15-19

[16] Montalescot G, Sechtem U, Achenbach S et al. 2013 ESC guidelines on the management of stable coronary artery disease: the Task Force on the management of stable coronary artery disease of the European Society of Cardiology. Eur Heart J 2013; 34: 2949-3003

[17] GBD 2017 Causes of Death Collaborators. Global, regional, and national age-sex-specific mortality for 282 causes of death in 195 countries and territories, 1980-2017: a systematic analysis for the Global Burden of Disease Study 2017. Lancet 2018 ; 392: 1736-1788

[18] Tousoulis D. What have we learned about using aspirin for primary prevention from the ASCEND and ARRIVE trials? Cardiovasc Res 2019; 115: e15-e16 
[19] Gaziano JM, Brotons C, Coppolecchia R et al. Use of aspirin to reduce risk of initial vascular events in patients at moderate risk of cardiovascular disease (ARRIVE): a randomised, double-blind, placebo-controlled trial. Lancet 2018; 392: 10361046

[20] McNeil J], Woods RL, Nelson MR et al. Effect of Aspirin on Disability-free Survival in the Healthy Elderly. N Engl J Med 2018; 379: $1499-1508$

[21] McNeil J], Wolfe R, Woods RL et al. Effect of Aspirin on Cardiovascular Events and Bleeding in the Healthy Elderly. N Engl J Med 2018; 379: 1509-1518

[22] McNeil J], Nelson MR, Woods RL et al. Effect of Aspirin on AllCause Mortality in the Healthy Elderly. N Engl J Med 2018; 379: 1519-1528

[23] Ibanez B, James S, Agewall S et al. 2017 ESC Guidelines for the management of acute myocardial infarction in patients presenting with ST-segment elevation: The Task Force for the management of acute myocardial infarction in patients presenting with ST-segment elevation of the European Society of Cardiology (ESC). Eur Heart J 2018; 39: 119-177

[24] Rothwell PM, Cook NR, Gaziano JM et al. Effects of aspirin on risks of vascular events and cancer according to bodyweight and dose: analysis of individual patient data from randomised trials. Lancet 2018; 392: 387-399

[25] Abdelaziz H, Saad M, Pothineni N et al. Aspirin for primary prevention of cardiovascular events. I Am Coll Cardiol 2019; 73: 2915-2929

[26] Gachet C. P2 receptors, platelet function and pharmacological implications. Thromb Haemost 2008; 99: 466-472

[27] Ittaman SV, VanWormer J], Rezkalla SH. The role of aspirin in the prevention of cardiovascular disease. Clin Med Res 2014; 12: $147-154$

[28] Randomised trial of intravenous streptokinase, oral aspirin, both, or neither among 17,187 cases of suspected acute myocardial infarction: ISIS-2. ISIS-2 (Second International Study of Infarct Survival) Collaborative Group. Lancet 1988; 2: 349360

[29] Baigent C, Blackwell L, Collins R et al. Aspirin in the primary and secondary prevention of vascular disease: collaborative meta-analysis of individual participant data from randomised trials. Lancet 2009; 373: 1849-1860

[30] Catalano M, Born G, Peto R. Prevention of serious vascular events by aspirin amongst patients with peripheral arterial disease: randomized, double-blind trial. J Intern Med 2007; 261: 276-284

[31] Aboyans V, Ricco JB, Bartelink MEL et al. 2017 ESC Guidelines on the Diagnosis and Treatment of Peripheral Arterial Diseases, in collaboration with the European Society for Vascular Surgery (ESVS): Document covering atherosclerotic disease of extracranial carotid and vertebral, mesenteric, renal, upper and lower extremity arteriesEndorsed by: the European Stroke Organization (ESO)The Task Force for the Diagnosis and Treatment of Peripheral Arterial Diseases of the European Society of Cardiology (ESC) and of the European Society for Vascular Surgery (ESVS). Eur Heart J 2017; 39: 763-816

[32] CAPRIE Steering Committee. A randomised, blinded, trial of clopidogrel versus aspirin in patients at risk of ischaemic events (CAPRIE). CAPRIE Steering Committee. Lancet 1996; 348: 1329-1339

[33] Schunkert H, Boening A, von Scheidt M et al. Randomized trial of ticagrelor vs. aspirin in patients after coronary artery bypass grafting: the TiCAB trial. Eur Heart J 2019; 40: 2432-2440
[34] Mehta SR, Yusuf S, Peters RI et al. Effects of pretreatment with clopidogrel and aspirin followed by long-term therapy in patients undergoing percutaneous coronary intervention: the PCI-CURE study. Lancet 2001; 358: 527-533

[35] Chen ZM, Jiang LX, Chen YP et al. Addition of clopidogrel to aspirin in 45,852 patients with acute myocardial infarction: randomised placebo-controlled trial. Lancet 2005; 366: 1607-1621

[36] Bhatt DL, Fox KA, Hacke W et al. Clopidogrel and aspirin versus aspirin alone for the prevention of atherothrombotic events. N Engl J Med 2006; 354: 1706-1717

[37] Bhatt DL, Flather MD, Hacke W et al. Patients with prior myocardial infarction, stroke, or symptomatic peripheral arterial disease in the CHARISMA trial. J Am Coll Cardiol 2007; 49: 1982-1988

[38] Bonaca MP, Bhatt DL, Cohen M et al. Long-Term Use of Ticagrelor in Patients with Prior Myocardial Infarction. N Engl J Med 2015; 372: 1791-1800

[39] Vranckx P, Valgimigli M, Jüni $P$ et al. Ticagrelor plus aspirin for 1 month, followed by ticagrelor monotherapy for 23 months vs. aspirin plus clopidogrel or ticagrelor for 12 months, followed by aspirin monotherapy for 12 months after implantation of a drug-eluting stent: a multicentre, open-label, randomised superiority trial. Lancet 2018; 392: 940-949

[40] Mehran R, Baber U, Sharma SK et al. Ticagrelor with or without Aspirin in High-Risk Patients after PCI. N Engl J Med 2019; 381: 2032-2042

[41] Anand SS, Bosch J, Eikelboom JW et al. Rivaroxaban with or without aspirin in patients with stable peripheral or carotid artery disease: an international, randomised, double-blind, placebo-controlled trial. Lancet 2018; 391: 219-229

[42] Piepoli MF, Hoes AW, Agewall S et al. 2016 European Guidelines on cardiovascular disease prevention in clinical practice: The Sixth Joint Task Force of the European Society of Cardiology and Other Societies on Cardiovascular Disease Prevention in Clinical Practice (constituted by representatives of 10 societies and by invited experts)Developed with the special contribution of the European Association for Cardiovascular Prevention \& Rehabilitation (EACPR). Eur Heart J 2016; 37: 23152381

[43] Vandvik PO, Lincoff AM, Gore JM et al. Primary and secondary prevention of cardiovascular disease: Antithrombotic Therapy and Prevention of Thrombosis, 9th ed: American College of Chest Physicians Evidence-Based Clinical Practice Guidelines. Chest 2012; 141 (Suppl. 2): e637S-e6685

[44] American Diabetes Association. 2. Classification and Diagnosis of Diabetes: Standards of Medical Care in Diabetes-2019. Diabetes Care 2019; 42 (Suppl. 1): S13-S28

[45] Arnett DK, Blumenthal RS, Albert MA et al. 2019 ACC/AHA Guideline on the Primary Prevention of Cardiovascular Disease: A Report of the American College of Cardiology/American Heart Association Task Force on Clinical Practice Guidelines. Circulation 2019; 140: e596-e646

Bibliografie

Kardiologie up2date 2020; 16: 315-328

DOI 10.1055/a-0917-8604

ISSN 1611-6534

(c) 2020. Thieme. All rights reserved.

Georg Thieme Verlag KG, Rüdigerstraße 14,

70469 Stuttgart, Germany 


\section{Punkte sammeln auf GME. thiemede}

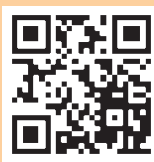

Diese Fortbildungseinheit ist in der Regel 12 Monate online für die Teilnahme verfügbar.

Den genauen Einsendeschluss finden Sie unter https://cme.thieme.de.

Sollten Sie Fragen zur Online-Teilnahme haben, finden Sie unter https://cme.thieme.de/hilfe

eine ausführliche Anleitung. Wir wünschen viel Erfolg beim Beantworten

der Fragen!

Unter https://eref.thieme.de/CXD5K12 oder über den QR-Code kommen Sie direkt zur Startseite des Wissenstests.

VNR 2760512020158721858

\section{Frage 1}

Welcher pharmazeutischen Wirkungsklasse wird ASS zugeordnet?
A Fibrinolytikum
B Thrombozytenaggregationshemmer
C Antihistaminikum
D Serotonin-Reuptake-Inhibitor
E Antibiotika

\section{Frage 2}

Welche Patientengruppe wurde in der ARRIVE-Studie hinsichtlich einer Primärprävention mit ASS untersucht?
A Patienten mit einem mittleren kardiologischen Risikoprofil
B ältere Menschen über 70 Jahre
C Diabetiker mit einem Mindestalter von 40 Jahren
D Parkinson-Patienten
E Patienten mit einer Herzinsuffizienz NYHA IV

\section{Frage 3}

Welche Nebenwirkung tritt am häufigsten unter ASS-Einnahme auf?
A Agranulozytose
B Tremor
C gastrointestinale Blutung
D anaphylaktischer Schock
E Übelkeit

\section{Frage 4}

Welche Komplikation wird vor allem bei älteren, sturzgefährdeten Patienten > 65 Jahre unter ASS-Einnahme verstärkt?
A kranielle Blutung
B Gastritis
C Fraktur des Femurs
D Schwindel
E Obstipation

\section{Frage 5}

Welche Studie untersuchte den Effekt von ASS in der Primärprävention auf Diabetes mellitus?
A ASPREE
B ASCEND
C ARRIVE
D „Effects of aspirin on risks of vascular events and cancer ac- cording to bodyweight and dose“
E ESC/ESAC-Leitlinien

\section{Frage 6}

Welcher Risikofaktor wurde nicht in der ARRIVE-Studie eingeschlossen?
A hoher Cholesterinwert (>200 mg/dl bei Männern/> $240 \mathrm{mg}$ bei Frauen)
B LDL (> $130 \mathrm{mg} / \mathrm{dl}$ bei Männern/> $160 \mathrm{mg} / \mathrm{dl}$ bei Frauen)
C niedriges $\mathrm{HDL}(<40 \mathrm{mg} / \mathrm{dl})$
D Rauchen
E Osteoporose

\section{Frage 7}

Auf welchem Mechanismus beruht die pharmakologische Wirkung von ASS?
A Hemmung der Cyclooxygenase 1 - COX 1
B Aktivierung des Renin-Angiotensin-Aldosteron-Systems
C Fibrinolyse
D Phosphorylierung des Glukosemoleküls
E Azetylierung eines Serinrestes

\section{Frage 8}

Wie lange wird die Thrombozytenaggregation durch ASS gehemmt?
A 30 Minuten
B 6 Stunden
C 5-12 Tage
D 4 Wochen
E 24 Stunden

- Weitere Fragen auf der folgenden Seite... 


\section{Punkte sammeln auf CME. thieme.de}

Fortsetzung...

\section{Frage 9}

Wie wirkt das Medikament ASS nicht?
A antipyretisch
B antiinflammatorisch
C analgetisch
D antithrombotisch
E spasmolytisch

\section{Frage 10}

Wie viel mg ASS wurde der ASS-Gruppe in der ARRIVE-Studie täglich verabreicht?
A $80 \mathrm{mg} / \mathrm{d}$
B $325 \mathrm{mg} / \mathrm{d}$
C $500 \mathrm{mg} / \mathrm{d}$
D $100 \mathrm{mg} / \mathrm{d}$
E $1000 \mathrm{mg} / \mathrm{d}$ 